\title{
Antimicrobial resistance and use in Canada: A federal framework for action
}

\author{
Public Health Agency of Canada*, in participation with the Canadian Food Inspection Agency, Canadian \\ Institutes of Health Research, Health Canada, and Agriculture and Agri-Food Canada
}

*Correspondence to: Lindsay.noad@phac-aspc.gc.ca

\begin{abstract}
Antimicrobial resistance (AMR) is a serious and growing global public health threat. Modern medical and veterinary practice depends on the widespread availability of effective antimicrobials to prevent and treat infections in humans and animals. Addressing the growing threat of AMR in Canada is a shared responsibility; Antimicrobial Resistance and Use in Canada: A Federal Framework for Action serves as a starting point for a collaborative response. The goal of the Framework is: "To protect Canadians from the health risks related to antimicrobial resistance." It includes three pillars: Surveillance, Stewardship, and Innovation. The Framework identifies concrete Government of Canada actions to reduce the threat and impact of AMR. Equally important, it is a vehicle to engage partners and stakeholders in discussions on efforts that, together, can significantly increase the results of individual actions. Beyond the Government of Canada, provinces and territories, academia, animal and human health professionals, food production stakeholders, animal producer groups and farmers, as well as private industry each hold essential levers to reduce AMR. By continuing to work together, we will collectively achieve greater results in reducing the risks of antimicrobial resistance and protecting the health and safety of all Canadians.
\end{abstract}

\section{Introduction}

Antimicrobial resistance (AMR) is a serious and growing global public health threat. Governments and health organizations around the world are paying increasing attention to the significant threat that this problem poses to modern medicine and the health of the global population. Antimicrobial Resistance and Use in Canada: A Federal Framework for Action is a just released publication that represents the Government of Canada's response to the threat of AMR (1). It provides a cohesive and collaborative approach across federal departments with mandates to address and mitigate AMR. Within Canada, this Framework will serve as a starting point for engagement and mobilization of all who are accountable for action on antimicrobial resistance and use.

\section{Why is antimicrobial resistance a problem?}

Modern medical and veterinary practice depends on the widespread availability of effective antimicrobials to prevent and treat infections in both humans and animals. Without them, the ability to fight infectious disease is significantly impeded. Each year in Canada, more than 18,000 hospitalized patients acquire infections that are resistant to antimicrobials. Deaths directly related to Clostridium difficile alone have increased five-fold in the past decade $(2,3)$.

\section{Why are we taking action now?}

There are activities that can be undertaken and expanded upon that will protect Canadians from the threat of AMR. However, with the increasing burden of antimicrobial resistance, the time to act is now. Domestic action is mirrored by a global call for action; World Health Assembly Member States endorsed a resolution that identified "the urgent need of a Global Action Plan for antimicrobial resistance" (4). Canada's Framework for Action represents an important step in expanding domestic efforts and responding to the global call for action on antimicrobial resistance. 


\section{A shared responsibility}

Addressing the growing threat of AMR in Canada is a shared responsibility. The Government of Canada's role in protecting Canadians against disease threats of national concern is essential to multi-sector collaboration. The federal role for AMR is included in the mandate of several departments: Public Health Agency of Canada (PHAC), Health Canada (HC), Canadian Food Inspection Agency (CFIA), Canadian Institutes of Health Research (CIHR), and Agriculture and Agri-food Canada (AAFC). Provinces and territories play a key role because they are responsible for the delivery of health care, approval of antimicrobials for medical coverage, and the regulation of antimicrobial use in agriculture and veterinary medicine. Professional and non-governmental organizations are also actively involved in addressing antimicrobial resistance and use.

\section{The Framework}

The goal of the Framework for Action is: "To protect Canadians from the health risks related to antimicrobial resistance." The federal government will take action and work with its partners to reduce the health risks associated with AMR in three areas: Surveillance, Stewardship, and Innovation.

\section{Surveillance}

Surveillance systems collect data and information that is used to protect the health of human and animal populations. Antimicrobial surveillance information informs effective antimicrobial resistance and antimicrobial use programs, guidelines, and policies.

\section{ACTION 1: Establish and strengthen surveillance systems to identify new threats or changing patterns in antimicrobial resistance and use, in human and animal settings.}

Canada has well-established surveillance systems for antimicrobial resistance and use. The Canadian Antimicrobial Resistance Surveillance System (CARSS) will be created, building on the foundation of the Public Health Agency of Canada's current antimicrobial resistance surveillance systems. CARSS will integrate available antimicrobial resistance data, clearly articulate and track antimicrobial resistance at a national level, and expand surveillance activities at the hospital and community level.

CFIA, AAFC, HC, and PHAC collaborate on the Canadian Integrated Program for Antimicrobial Resistance Surveillance (CIPARS). Moving forward this work will be strengthened and linked to CARSS. For example, CFIA and AAFC are planning increased surveillance on antimicrobial use in animal settings to ensure that a comprehensive data set is available through CIPARS.

These and other actions will provide a broader understanding of antimicrobial use and resistance across human populations in hospital and in the community, and animals in veterinary and agricultural settingsall in support of keeping antimicrobials effective.

\section{Stewardship}

Conserving the effectiveness of currently available antimicrobials is vital in mitigating the threat posed by antimicrobial resistant microbes. Public awareness activities help Canadians understand the benefits and risks of antimicrobials. The public plays a key role in infection prevention through day-to-day practices such as good hand hygiene. 


\section{ACTION 2: Strengthen the promotion of the appropriate use of antimicrobials in human and veterinary medicine.}

The Government of Canada will engage the public through an Antimicrobial Resistance Awareness Campaign on antimicrobial use and infection control during Antibiotic Awareness Week 2014 (November 17-21). Future awareness activities will build on lessons learned from these activities. Health Canada is working to increase veterinary oversight of the use of medically important antimicrobials in food animal production. Efforts are also currently underway to phase out growth promotion claims on medically important antimicrobials.

ACTION 3: Work with the animal agriculture sector partners to strengthen the regulatory framework on veterinary medicines and medicated feeds, including facilitating access to alternatives, and encourage the adoption of practices in order to reduce the use of antimicrobials.

The Government of Canada's role in the regulation of animal health includes the sale of veterinary drugs, medicated feeds, and vaccines. Its regulatory activities present an opportunity to further advance stewardship and the adoption of best practices in Canada. Through legislative and regulatory authorities, the Government can engage with those who must comply with the regulations to identify innovative approaches to facilitate access to alternatives.

\section{Innovation}

Innovation fosters new methods and tools that combat AMR and improve antimicrobial use. Research informs health services and policy through innovative solutions, ranging from new or alternative antimicrobials and therapies, through faster and more accurate diagnostics. The federal government supports ongoing domestic health research and innovation while collaborating with international partners to contribute to global research efforts on antimicrobial resistance, antimicrobial use, novel therapies, and alternative antimicrobials.

\section{ACTION 4: Promote innovation through funding collaborative research and development efforts on antimicrobial resistance both domestically and internationally.}

The Government of Canada is committed to increasing its research investment to support innovation. Through the CIHR-funded Canada-U.K. partnership on antimicrobial resistance, and other CIHR-funded research projects, research is currently underway to better understand the nature of resistance, investigate novel therapies, identify alternatives to antimicrobials, develop diagnostic tools, and find new ways of using existing antimicrobials.

\section{Moving forward}

The Framework identifies concrete Government of Canada actions to reduce the threat and impact of antimicrobial resistance. Equally important, it is a vehicle to engage partners and stakeholders in discussions on efforts that, together, can significantly increase the results of individual actions. Beyond the Government of Canada, provinces and territories, academia, animal and human health professionals, food production stakeholders, animal producer groups and farmers, as well as private industry each hold essential levers to reduce antimicrobial resistance. Working in collaboration will support efforts across both human and animal health settings.

\section{Conclusion}

Canada is already taking significant action to address the threat of AMR both domestically and internationally. By continuing to work together, we will collectively achieve greater results in reducing the risks of antimicrobial resistance and protecting the health and safety of all Canadians. 


\section{Acknowledgements}

Many thanks to the PHAC AMR Tiger Team and collaborators at: Health Canada, Canadian Food Inspection Agency, Canadian Institutes of Health Research, and Agriculture and Agri-Food Canada.

\section{Conflict of interest}

None.

\section{References}

(1) Public Health Agency of Canada. Antimicrobial resistance and use in Canada: A federal framework for action. http://healthycanadians.gc.ca/drugs-products-medicaments-produits/antibiotic-resistanceantibiotique/antimicrobial-framework-cadre-antimicrobiens-eng.php

(2) Public Health Agency of Canada (PHAC). Healthcare-associated Clostridium difficile infections in Canadian acute-care hospitals: Surveillance report January 1st, 2007 to December 31st, 2012. Ottawa: Centre for Communicable Diseases and Infection Control, PHAC; 2014.

http://www.ammi.ca/media/69406/CDI\%20Surveillance\%20Report\%20Jan\%202007\%20to\%20Dec\%202012\%20 Final_Eng\%20Aug\%2025\%202014.pdf

(3) Gravel D, Miller M, Simor A, Taylor G, Gardam M, McGeer A, Hutchinson J, Moore D, Kelly S, Boyd D, Mulvey M, Bryce E, Conly J, Dow G, Embil J, Embree J, Forgie S, Frenette C, Henderson E, John M, Johnston L, Kibsey $\mathrm{P}$, Langley J, Loe. Health care-associated Clostridium difficile infection in adults admitted to acute care hospitals in Canada: A Canadian nosocomial infection surveillance program study. Clinical Infectious Diseases. 2009; 48(5):568-76.

(4) World Health Organization (WHO). Antimicrobial drug resistance-Report by the Secretariat. WHO: 2014 Mar 14. http://apps.who.int/gb/ebwha/pdf_files/WHA67/A67_39-en.pdf 